\section{Respiratory fluoroquinolones} vs. other commonly used antimicrobials in

mild-to-moderate severity community-acquired pneumonia

\section{Abstract}

Objective: Respiratory fluoroquinolones (RFQs) are widely used in the treatment of community-acquired pneumonia (CAP) in our region. Our aim was to find if there was outcome difference between RFQ-based versus RFQ-exempt regimens.

Methods: A retrospective study of RFQs versus other used antimicrobial therapy (OUAT) in the treatment of patients with mid-to-moderate CAP adjusted by pneumonia severity score (PSI). Rates of treatment outcome at end-of- therapy i.e. clinical improvement, length of hospital stay and speed of recovery were evaluated. Patients were included if they had Mild-to-Moderate severity CAP, $\geq 18$ years old, completed $\geq 3$ days of antimicrobials.

Results: 320 patients were included, mean age for all groups was 49.63 years $(P=0.204)$, males $60.3 \%(P=0.219)$. All had similar PSI score (Pearson $X^{2}$ test $\left.=13.75, P=0.185\right)$. The first group $(24.4 \%)$ is composed of RFQs monotherapy. The second group (50.6\%) is composed of RFQs plus B-lactams. The third group (25\%) is composed of OUAT. Diabetes was the most common comorbidity among all ( $P=$ $0.847)$. There was no significant difference among the three groups in clinical improvement $(P=0.424)$ and speed of recovery $(P=0.398)$, however length of hospital stay was significantly shorter for the RFQs monotherapy $(P=0.004)$.

Cumulative curve for probability of discharge did not show significant difference among the three therapy groups $(P \geq 0.20)$
Jamal Wadi Al Ramahi MD, FIDSA $A^{1,2}$, Ahmad Al Sayed Pharm D. $\mathrm{MSc}^{3}$, Ayham Nassar MD2, Mais F. Kadri M.D', Omar Al-Eter M.D', Hiba F.Nour M.D'1, Muhannad Qulaghassi MD², Nader Abdel Jawad MD², Dareen Smaik, MD', Sahar Al Shair Pharm D ${ }^{3}$

1 Jordan Hospital and Medical Center. Amman - Jordan, The Specialty Hospital. Amman - Jordan, Arab Medical Center. Amman - Jordan

Corresponding author:

Jamal Wadi MD, FIDSA

$\equiv$ jamalwadimd@yahoo.com 
Conclusion: There were no significant difference among the patient groups regarding end of treatment and clinical improvement rates, speed of recovery and probability of hospital discharge. However, they significantly differ in length of hospital stay for RFQs monotherapy.

Keywords: Respiratory quinolones, quinolones, Community-acquired pneumonia, length of hospital stay, 30 days mortality, speed of recovery.

\section{Introduction}

Community-acquired pneumonia is one of the most common infections afflicting individuals, each year about four millions are diagnosed with CAP in USA, one-quarter are hospitalized with considerable morbidity and mortality. It is arbitrarily classified into mild, moderate and severe, whether for outpatient therapy, in-hospital, or ICU. Severity is classified based on either CURB 65 (confusion, uremia, respiratory rate, low blood pressure, age $\geq 65$ years) or pneumonia severity index (PSI) which is more sophisticated but a more accurate tool. CAP incidence ranges between 12 cases per 1,000 population -year to as high as 18.2, 27.9, 52.3 case per 1000 population-year in individuals aged 65 - 69, $75-79$ and $\geq 85$ years respectively $[1,2,3,4]$.

Due to these inevitabilities, caring for adult patients with CAP has been the focus of many guidelines such as the Infectious Diseases Society of America/ American Thoracic Society (IDSA/ATS) and the British Thoracic Society (BTS) among other societies. Studies addressing the outcome difference among various classes of antimicrobial agents prescribed in the treatment of CAP were conducted where quinolones were found superior to the comparator antimicrobials. In a meta-analysis comparing different quinolones including levofloxacin, gemifloxacin and moxifloxacin, quinolones' treated patients were found to have better clinical and microbiological response compared to macrolides and B-lactams, in addition, they were found more effective in severe pneumonia, those in need of hospitalization and parenteral therapy [5]. Furthermore, some studies compared the treatment outcome among quinolones; Olivier Leroy et al. compared ofloxacin or ciprofloxacin plus B-lactam with a newer quinolone i.e. levofloxacin plus a B-lactam. They concluded that levofloxacin is associated with lower mortality than ofloxacin or ciprofloxacin in severe pneumococcal CAP [6]. However, the British thoracic society guidelines statement; quinolones were found not superior to B-lactams, macrolides in combination with B-lactams and B-lactam B-lactamase inhibitors in the treatment of patients with mild-to-moderate severity CAP, also due to their higher cost and association with methicillin resistant staphylococcus aureus (MRSA) and Clostridium difficile infection (CDI) they are not preferred as first line agents [7].

In our study, we examine if there are differences among using RFQ monotherapy versus combination, and other antimicrobial regimens not containing RFQs in patients with Mild-to-Moderate CAP requiring admission to a hospital ward. The primary outcome measures were clinical response at end of therapy and speed of recovery. Secondary outcome measures were mortality by 30 days and length of hospital stay. 


\section{Materials and Methods}

\section{Study structure and site}

A multicenter retrospective study in three hospitals; a community service and two teaching hospitals; combined encompass about 750 beds. Data were collected in retrospective manner in between September 2012 - April 2014. Patients were included if they were in hospital ward, Mild-to-Moderate severity CAP, received oral or parenteral therapy, on RFQs, RFQs in combination with B-lactams, on macrolides/ß-lactams combination, on B-lactam monotherapy, on macrolides monotherapy or other antimicrobial agents utilized in CAP, were $\geq 18$ years old, who completed $\geq$ 3 days of the study antimicrobial and isolated bacteria is/are susceptible to at least $\geq 1$ antimicrobial agent should a culture was available. Patients were excluded if they had health care associated pneumonia (HCAP), CAP required ICU admission, and CAP treated as an out patient, pregnancy, and $<18$ years of age and lung cancer. Patients were contacted by telephone 30 days after their hospital discharge. The primary outcome measures were rates of clinical improvement at end of therapy and speed of recovery, secondary outcome measures were mortality by 30 days and length of hospital stay.

\section{CAP Definition}

Patients who suffer of symptoms and signs of CAP including cough, fever, chills, rigors, chest pain, pleurisy, dyspnea and sputum production (mucopurulent, scant or watery), gastrointestinal symptoms including nausea, vomiting, diarrhea, and mental status changes, respiratory rate $\geq 24$ breaths/minute, tachycardia, audible rales or bronchial sounds.

Laboratory evaluation: leukocytosis with a left shift or leukopenia. The presence of an infiltrate on a plain chest radiograph or a chest $C T$ is considered the "gold standard" for diagnosing pneumonia when clinical and microbiologic features are supportive. Microbiological diagnosis includes an accepted sputum specimen for Gram's stain and or culture [8], urinary antigen testing, blood culture is reliable if positive and establishes the microbial diagnosis in the context of diagnosing CAP [4].

\section{Statistical methods}

Statistical analysis was set using SPSS version 18 statistical software. Continuous variables were analyzed for significant differences by ANOVA, $\alpha$-significance ( $P$-value) is considered significant for differences if $<0.05$. If significant difference was detected among groups, sensitivity analysis by Tuckey HSD test among variables was performed. Pearson Chi Square $\left(\chi^{2}\right)$ was used to detect significant differences among comorbidities, laboratory, and radiological diagnoses among different antimicrobial therapy groups. Frequencies were used where appropriate for nominal variables. Cumulative curves for the probability of hospital discharge were plotted for mild CAP (Figure 1); representative curves were

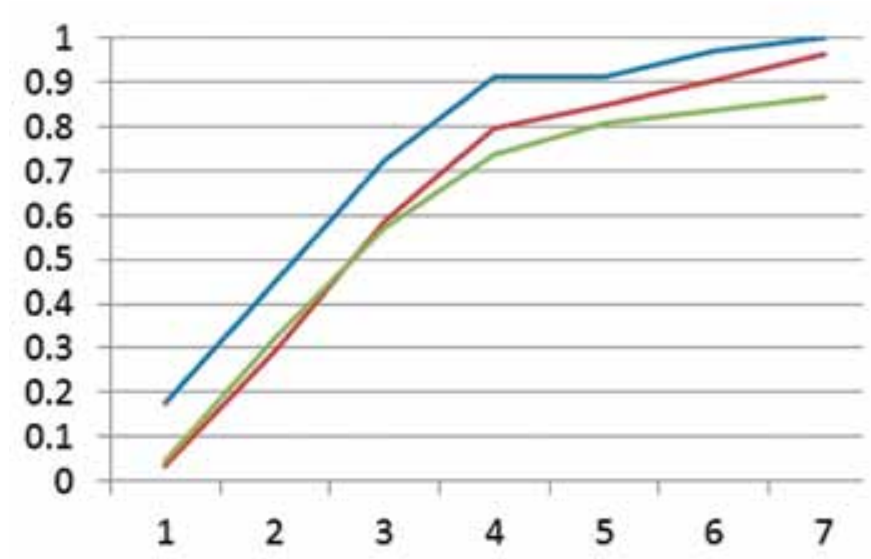

Figure 1. Cumulative Probability Curve for Hospital Discharge in Patients with Mild CAP* in the Therapy Groups

P-values were tested by Kolmogorov-Smirnov test using RFQs as the reference curve

RFQs: Respiratory quinolounes. RFQs+: RFQs in combination with B-lactams .OUAT: Other commonly used antimicrobial agents for CAP

*The majority of patients were in mild CAP $(N=276$; RFQs $=69$, RFQ+ other agents = 139, OUAT =68), 43 patients were in medium severity group and 1 no PSI available data. 


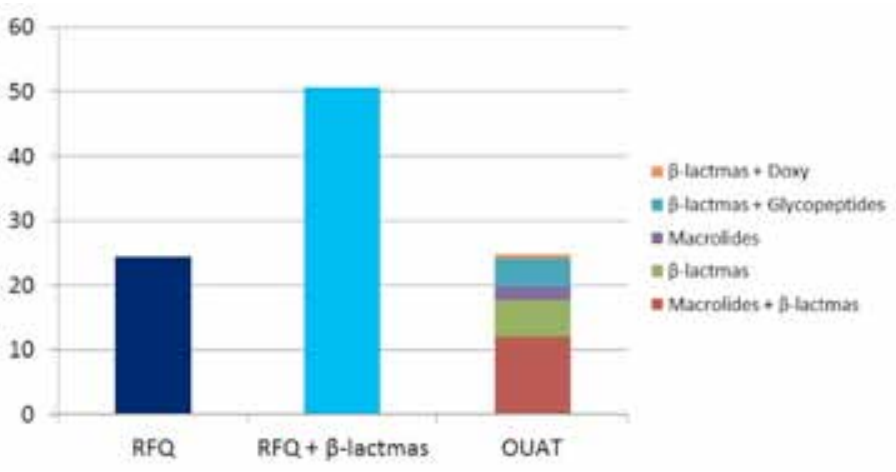

Figure 2. Ratios of Antimicrobials Therapy Used to Treat Patients with Mild-to-Moderate Community-Acquired Pneumonia

The three therapy groups had no statistical significant PSI score difference; Pearson $\mathrm{X}^{2}$ test $=13.75, p=0.185$

RFQ: Levofloxacin or Moxifloxacin; Doxy: Doxycycline; Macrolides: Azithromycin or Clarithromycin; Glycopeptides: Teicoplanin or Vancomycin; B-lactmas: Aminopeicillins, $3 r^{d}$ Generation Cephalosporin or Carbapenems.

tested for significant differences for the first seven days using RFQs curve as the reference curve for the other two curves by Kolmogorov-Smirnov test using a web application. Found on http://scistatcalc. blogspot.com/2013/11/kolmogorov-smirnov-testcalculator.html.

\section{Results and Discussion}

A total of 330 patients were included in the study, of these 10 were excluded; 1 pregnant, 1 ICU-requiring admission, 2 insufficient data and 6 patients for not having the diagnosis of CAP and were not treated as such. The mean age for the three antimicrobial therapy groups combined was 49.63 years, each group age mean has no significant difference from the common mean $(P=0.204)$, the same was for gender distribution, males $60.3 \%(P=0.219)$. The three groups had similar patients' distribution as scored by PSI (Pearson $X^{2}$ test $=13.75, P=0.185$ ). The first group is composed of RFQs (Levofloxacin or Moxifloxacin) monotherapy used in $24.4 \%$ of patients. The second group is composed of a RFQ combined with B-lactams in $50.6 \%$ of patients. The third group (25\%) is composed of the rest; macrolides (Azithromycin or Clarithromycin) combined with B-lactams in $12.2 \%$ of patients, B-lactams monotherapy (5.7\%), macrolides monotherapy 2.1\%, glycopeptides (11 teicoplanin 3 vancomycin) with B-lactams $4.4 \%$ and Doxycycline combined with B-lactams (2 patients) $0.6 \%$ (Figure 2).

Comorbidities had no significant distribution difference among the three groups as shown in ( $\mathrm{Ta}$ ble 3). Diabetes mellitus was the most common comorbidity, a total of 84 patients almost evenly spread $(P=0.847)$, followed by kidney disease (17 patients), bronchial asthma (15 patients), COPD (11 patients), and immunosuppression (13 patients), whereas other comorbidities had no significant differences as well $(P>0.05)$. Radiological diagnosis by plain X-rays and CT-scan was universal among patients. The percentage-yield of the radiological diagnoses showed no significant difference among the three therapy groups for plain $X$-Rays ( $P=0.447$ ) and for CT-Scan $(P=0.247)$. Though modest frequencies of symptoms were obtained from records like (Fever, sputum, chest pain, chills/rigors, cough, shortness of breath, vomiting and other symptoms), yet all groups did not show statistically significant differences (Table 1). Sputum culture was minimally utilized; it was requested in 39 out of 320 patients and a microorganism was identified twice; one Streptococcus pneumoniae and the other was Haemophilus influenzae. Blood cultures were ordered 34 times although febrile patients counted 202 on admission; but only one $S$. aureus was identified.

Rates of clinically improved patients had no significant difference among the three therapy groups $(P=0.424)$, as well as speed of recovery $(P=0.398)$, however rates of length of hospital stay was significantly shorter for the RFQs monotherapy group $(P=0.004)$, even higher significance was detected for mild CAP among the three groups $(P<0.0001)$. Mortality occurred in four patients; 1 patient in the 
Table 1. Demography and characteristics of 320 patients with mild-moderate CAP.

\begin{tabular}{|c|c|c|c|c|c|}
\hline \multirow{2}{*}{\multicolumn{2}{|c|}{ Characteristics }} & \multicolumn{2}{|c|}{ RFQs } & \multirow{2}{*}{ OUAT } & \multirow{2}{*}{ P-value* } \\
\hline & & Monotherapy & Combination \& & & \\
\hline Age mean \& (No. of included patients) & $\begin{array}{r}\text { Male } \\
\text { Female }\end{array}$ & $\begin{array}{l}49.62(45) \\
57.09(33)\end{array}$ & $\begin{array}{c}48.23(104) \\
47.77(66)\end{array}$ & $\begin{array}{l}48.27(45) \\
51.42(38)\end{array}$ & $\begin{array}{l}0.97 @ \\
0.14 @\end{array}$ \\
\hline Gender: N and (\%) & $\begin{array}{l}\text { Male } \\
\text { Female }\end{array}$ & $\begin{array}{l}45(22.7) \\
33(25.2)\end{array}$ & $\begin{array}{c}105(53) \\
57(43.5)\end{array}$ & $\begin{array}{c}45(22.7) \\
38(29)\end{array}$ & 0.534 \\
\hline $\begin{array}{l}\text { Comorbidity (No.) } \\
\text { Bronchial asthma } \\
\text { COPD } \\
\text { Diabetes mellitus } \\
\text { Kidney disease } \\
\text { Immunosuppression } \$ \\
\text { Smoking } \\
\text { Others } \#\end{array}$ & & $\begin{array}{c}3 \\
6 \\
21 \\
1 \\
4 \\
18 \\
23\end{array}$ & $\begin{array}{c}7 \\
3 \\
41 \\
10 \\
6 \\
16 \\
55\end{array}$ & $\begin{array}{c}5 \\
2 \\
23 \\
6 \\
3 \\
49 \\
36\end{array}$ & $\begin{array}{l}0.737 \\
0.058 \\
0.847 \\
0.172 \\
\text { NS } \\
>0.147 \\
\text { NS }\end{array}$ \\
\hline $\begin{array}{c}\text { Number and percentage of radiological } \\
\text { Plain X-ray } \\
\text { CT scan }\end{array}$ & agnosis (\% & $\begin{array}{l}73.0 \\
21.8\end{array}$ & $\begin{array}{l}80.2 \\
32.0\end{array}$ & $\begin{array}{c}78.75 \\
27.5\end{array}$ & $\begin{array}{l}0.447 \\
0.247\end{array}$ \\
\hline $\begin{array}{l}\text { Symptoms in patients } \\
\text { diagnosed with CAP (\%) } \\
\text { Fever } \\
\text { Sputum } \\
\text { Chest pain } \\
\text { Chills/Rigors } \\
\text { Cough } \\
\text { Shortness of Breath } \\
\text { Vomiting } \\
\text { Other Symptoms }\end{array}$ & & $\begin{array}{l}60.3 \\
34.6 \\
23.1 \\
19.2 \\
82.1 \\
42.3 \\
2.6 \\
35.9\end{array}$ & $\begin{array}{c}65.4 \\
50 \quad 108 \\
25.3 \\
24.1 \\
82.1 \\
50 \\
4.9 \\
29\end{array}$ & $\begin{array}{c}59 \\
31 \\
20.5 \\
21.7 \\
80.5 \\
50.6 \\
1.2 \\
22.9\end{array}$ & $\begin{array}{l}\text { NS } \\
\text { NS } \\
\text { NS } \\
\text { NS } \\
\text { NS } \\
\text { NS } \\
\text { NS } \\
\text { NS }\end{array}$ \\
\hline
\end{tabular}

\& In combination with B-lactams, B-lactams B-lactamases inhibitors, macrolides, glycopeptides, doxycycline or carbapenems (see text).

* Tested by Pearson $\chi^{2}$ test unless stated differently.

@ One way ANOVA, $\mathrm{F}=1.599$.

OUAT: Other used antimicrobial therapy in CAP, N: number

RFQs: Respiratory fluoroquinolones.

N/A: Not available

\# Others comorbidities: Coronary artery diseases, hypertension and one bronchiectasis in the RFQs. No alcoholism, asplenia, HIV and endobronchial obstruction were found.

$\$$ One hematological malignancy and one solid tumor (not lung) in RFQs, no patients were actively treated by immunosuppressive therapy at the time of CAP treatment. 
RFQs monotherapy group, and 3 patients in the RFQ- B-lactam combination group (Table 2).

Furthermore, Pearson Chi Square analysis was done for clarifying any possible difference in outcome between mild and moderate CAP for the three therapy subgroups, it showed no significant differences in rates of clinical improvement among the three therapy groups whether mild or moderate CAP $(P$ $=0.552)$, days to recover $(P=0.801$ for mild and 0.557 for moderate severity), death $(P>0.6)$, length of hospital stay showed no significant difference for moderate severity CAP $(P=0.717)$. However there was a highly significant difference in length of stay among the groups $(P<0.0001)$ for mild CAP in favor of RFQs (Table 2). Cumulative curve for the probability of discharge for the first seven days was constructed using RFQs probability of discharge curve as a reference to compare RFQs-in-combination and OUAT to; both were not significantly different as tested by Kolmogorov-Smirnov test, they were (Maximum deviation D $=0.428571$, KS statistic $=0.801784$ and $p=0.54)$ and $(D=0.571429$, KS statistic $=1.069045, P=0.20$ ) respectively.
CAP remains a commonly encountered infectious disease in clinical practice world-wide; its incidence is relatively high in all age groups. In our cohort of 320 patients study, we focus our attention on the most common adult Mild-to-Moderate CAP [1]. Here we sought to reveal whether RFQs monotherapy, commonly prescribed antimicrobials, differ in outcome (clinical improvement), speed of recovery and length of hospital stay from other regimens. RFQs used in this study were Levofloxacin and moxifloxacin, both in monotherapy and in the combination groups; Both RFQs were found clinically equivalent in Mild-to-Moderate CAP with similar safety profile. A previous study evaluated elderly patients $\geq$ 65 years old showed that moxifloxacin superseded levofloxacin in speed of recovery $(P=0.01)$ though clinical improvements at end of therapy were similar $[2,9,10]$.

The end-of- treatment recovery and speed of recovery for patients in the RFQs monotherapy versus other groups did not differ significantly, implying that in age-mixed population with mild-to-moderate $C A P$, the choice among the recommended an-

Table 2. Rates of Improvement in Patients@ Treated with RFQs monotherapy versus, RFQs in Combination and OUAT for Mild-to-Moderate Community-Acquired Pneumonia as Adjusted by PSI

\begin{tabular}{|c|c|c|c|c|}
\hline \multirow{2}{*}{ Outcome measure } & \multicolumn{2}{|c|}{ Respiratory Fluorquinolones } & \multirow[b]{2}{*}{ OUAT } & \multirow{2}{*}{ P-value* } \\
\hline & Monotherapy & Combination & & \\
\hline Clinically Improved (\%) & 97.4 & 93.2 & 100 & 0.424 \\
\hline Length of hospital stay (days)** & 2.92 & 3.91 & 3.77 & $0.004^{\#}$ \\
\hline Days to recovery & 2.31 & 2.31 & 2.08 & 0.398 \\
\hline Mortality by day $30^{\&}$ & 1.0 & 3.0 & 0.0 & -- \\
\hline
\end{tabular}

OUAT: Other Used Antimicrobial Therapy. PSI: Pneumonia severity score,

@ Patients number differ for each cell.

* Significance was tested by ANOVA, F-statistic

** By ANOVA; F = 5.65, Tukey HSD showed significant difference for RFQs versus OUAT $(p=0.044)$ and versus RFQs in combination ( $p=0.003$ ).

\# Highly significant difference was found for mild severity CAP $(p<0.0001)$.

\& one in severe PSI class in RFQs group and two in moderate and one in severe PSI class in combination therapy. 
timicrobials is not a major concern if one considers appropriate agents and cost effectiveness in the treatment of mild-moderate CAP .Nonetheless, the three groups did differ significantly in length of hospital stay in favor of RFQs monotherapy $(P=0.004)$, further analysis revealed that the difference became highly significant if mild CAP was solely compared $(P<0.0001)$; an interesting point in this study is that, if length of hospital stay is shorter, then we may conclude that speed of recovery should have been faster and patients were discharged sooner. Hitherto, this may have an impact on cost savings when treating mild CAP that require hospital-ward admission [11]. Cumulative probability of daily hospital discharge in the first week, though was numerically higher for the RFQs group, it did not reach statistical difference among the three therapy groups $(P>0.05)$ (Figure 1).

Nonetheless, due to the retrospective nature of the study and the use of different treatment protocols, one cannot make a firm conclusion. Shorter length of stay for the RFQs group monotherapy, especially in the mild CAP subset and not in RFQ-combination group may be due to the fact that patients were on parenteral B-lactams, and physicians taking care of patients may have been delinquent in discontinuing parenteral antimicrobials and replacing them with oral agents, having uncertainty about the agent that caused response in the absence of microbiological diagnosis. RFQs are popular for use among clinicians for their simple dosage format, patients' compliance, relatively low adverse effects, and the relatively low resistance profile in pneumococcal pneumonia despite high utilization [12]. Unfortunately, in the era of new virulent resistance patterns [13], an intense discussions about antimicrobial stewardship and prudent use of antimicrobials is ongoing $[14,15]$, many of practicing physicians have used unconventional, complex antimicrobial treatment regimens, including carbapenems and glycopeptides in combinations, at times with a macrolide or a quinolone. We can only hope that this study will convince physicians that simple recommended regimens in the treatment of Mild-to-Moderate CAP is as good as complicated unnecessary ones, with less patients' adverse effects [16], and promoting less resistance [17].

An added concern is that in real life, practicing physicians were not keen to obtain sputum for analysis and culture as well as blood cultures, though $32.8 \%$ of patients produced sputum and $63.1 \%$ had fever, unfortunately this attitude has been increasing worldwide over years $[18,19]$, though all patients needed hospital admission for more aggressive management.

\section{Acknowledgment}

We thank Reem Wadi AlRamahi BSc for her statistical assistance, and Waseem Abu Ashour PharmD, MSc School of Pharmacy, Memorial University of Newfoundland-Canada, for his comments.

Financial support and Conflict of interest: None

\section{References}

1. Patrick Petitpretz, Pierre Arvis, Miroslav Marel, Joaquim Moita, Juan Urueta; The CAP5 Moxifloxacin Study Group. Oral Moxifloxacin vs High Dose Amoxycillin in the Treatment of Mildto-Moderate Community-Acquired Suspected Pneumococcal Pneumonia in Adults. Chest 2001; 119:185-195

2. Antonio Anzueto, Michael S. Niederman, James Pearle, Marcos I. Restrepo, Albrecht Heyder, and Shurjeel H. Choudhri, for the Community-Acquired Pneumonia Recovery in the Elderly Study Group. Community-Acquired Pneumonia Recovery in the Elderly (CAPRIE): Efficacy and Safety of Moxifloxacin Therapy versus That of Levofloxacin Therapy. CID 2006; 42:73-81

3. Patrick P. Gleason et al. Association between Initial Antimicrobial Therapy and Medical Outcomes for Hospitalized Elderly Patients. Arch Intern Med. 1999; 159: 2562-2572.

4. Lionel A. Mandell, Richard G. Wunderink, Antonio Anzueto, John G. Bartlett, et al. Infectious Diseases Society of America/ American Thoracic Society Consensus Guidelines on the 
Management of Community-Acquired Pneumonia in Adults. CID 2007; 44:S27-72 )

5. Konstantinos Z. Vardakas, Ilias I. Siempos, Alexandros Grammatikos, Zoe Athanassa, et al. Respiratory fluoroquinolones for the treatment of community-acquired pneumonia: a metaanalysis of randomized controlled trials. CMAJ 2008; 179(12): 1269-77

6. David Olive, Hugues Georges, Patrick Devos, Nicolas Boussekey, Arnaud Chiche, et al. Severe pneumococcal pneumonia: impact of new quinolones on prognosis. BMC Infectious Diseases 2011; 11:66

7. 1W S Lim, S V Baudouin, R C George, et al. BTS guidelines for the management of community acquired pneumonia in adults: update 2009. Thorax 2009;64 (Suppl III):iii1-iii55

8. Stavros Anevlavis et al. A prospective study of the diagnostic utility of sputum gram stain in pneumonia. J Infect 2009; 59:83-89

9. Sun Tieying, Sun Li, Wang Rongmei, Ren Xiaoping, Sui Dongjiang, et al. Clinical efficacy and safety of moxifloxacin versus levofloxacin plus metronidazole for community-acquired pneumonia with aspiration factors. Chin Med J 2014;127(7):12011205

10. Joel Morganroth, John P. DiMarco, Antonio Anzueto, Michael S. Niederman, and Shurjeel Choudhri; for the CAPRIE Study Group. A Randomized Trial Comparing the Cardiac Rhythm Safety of Moxifloxacin vs Levofloxacin in Elderly Patients Hospitalized With Community-Acquired Pneumonia. Chest 2005; 128:3398-3406

11. Michael J Fine, Hugh M Pratt, D.Scott Obrosky, Judith R Lave, Laura J Mclntosh, et al. Relation between length of hospital stay and costs of care for patients with community-acquired pneumonia. Am J Med 2000;109(5):378-385

12. Pletz, M.W., et al., Low prevalence of fluoroquinolone resistant strains and resistance precursor strains in Streptococcus pneumoniae from patients with community-acquired pneumonia despite high fluoroquinolone usage. Int. J. Med. Microbiol. (2010), doi:10.1016/j.ijmm.2010.05.004

13. Imad M. Tleyjeh, Haytham M. Tlaygeh, Rana Hejal, Victor M. Montori, and Larry M. Baddour. The Impact of Penicillin Resistance on Short-Term Mortality in Hospitalized Adults with Pneumococcal Pneumonia: A Systematic Review and MetaAnalysis. CID 2006; 42:778-97

14. Ravina Kullar, Debra A. Goff, Lucas T Schulz, Barry C. Fox, and Warren E. Rose. The "Epic" Challenge of Optimizing Antimicrobial Stewardship: The Role of Electronic Medical Records and technology. CID 2013;57(7):1005-13

15. Charani, E. Castro-Sanchez, N. Sevdalis, Y. Kyratsis, L. Drumright, et al. Understanding the Determinants of Antimicrobial Prescribing Within Hospitals: The Role of 'Prescribing Etiquette". CID 2013;57(2):188-96

16. Carmen M. Faulkner, Heather L. Cox, and John C. Williamson. Unique Aspects of Antimicrobial Use in Older Adults. CID 2005; 40:997-1004
17. Christopher A. Ohl and Elizabeth S. Dodds Ashley. Antimicrobial Stewardship Programs in Community Hospitals: The Evidence Base and Case Studies. CID 2011;53(S1):S23-S28

18. John G. Bartlet. Diagnostic Tests for Agents of CommunityAcquired Pneumonia. CID 2011;52(S4):S296-S304.

19. Jamal Ahmad Wadi. Quinolones in Community-Acquired Pneumonia; Are they born Equal. IAJAA 2012;2(3:1). doi:10.3823/715

\section{Comment on this article:}

\section{f) 8 in $8+S$. P}

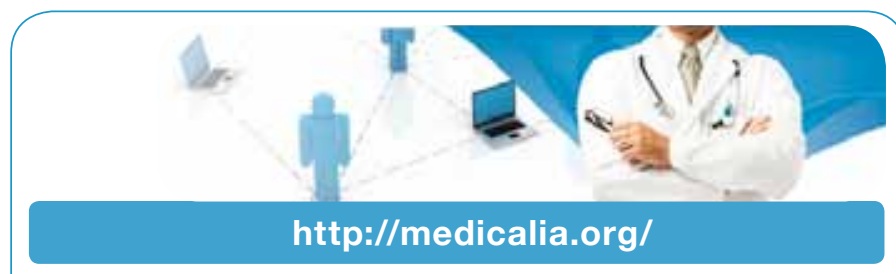

Where Doctors exchange clinical experiences, review their cases and share clinical knowledge. You can also access lots of medical publications for free. Join Now!

\section{Publish with iMedPub}

http://www.imed.pub

The Journal is an open access peer-reviewed journal that publishes scientific papers about all aspects of antimicrobials. The journal will publish original research articles, reviews, brief reports and case reports dealing with basic and clinical antibacterial agents, antiviral, antiprotozoals, antituberculuous, antifungal and antihelminthes agents. All manuscripts must be prepared in English, and are subject to a rigorous and fair peer-review process. Accepted papers will immediately appear online.

The journal aims to advance the knowledge, attitude and the research of chemotherapy in the Arabic world in cooperation with international, national scientific and public societies as well as research centers with similar aims and objectives.

\section{Submit your manuscript here:} www.iajaa.org 